\title{
L'Enseignement de l'Electronique Accessible aux étudiants Handicapés
}

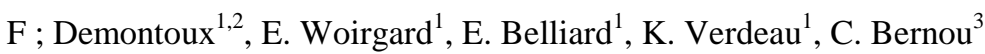 \\ francois.demontoux@iut.u-bordeaux1.fr \\ ${ }^{1}$ Université Bordeaux 1- cellule Phase \\ ${ }^{2}$ Université Bordeaux 1 - IUT Génie électrique et Informatique Industrielle \\ ${ }^{3}$ Université Bordeaux 1 - IUT Mesures Physiques
}

\begin{abstract}
RESUME : Une réflexion a été entamée au sein de l'équipe d'accueil des étudiants handicapés de l'Université de Bordeaux 1 (PHASE) afin de permettre à ces étudiants d'avoir la possibilité de réaliser les mêmes travaux pratiques que leurs camarades. Cela passe par un travail d'adaptation du poste de travail de l'étudiant et des maquettes de travaux pratiques.

Les métiers liés aux formations de l'EEA font de plus en plus appel à des outils qui permettent de créer des interfaces Homme-Machine. Les actions physiques complexes nécessaires à ces métiers se réduisent considérablement ce qui rend ces métiers accessibles à des personnes handicapées. Au-delà de notre mission de formation qui doit refléter cette évolution, nos universités ont aussi une mission d'information en direction des industriels afin de réduire leurs aprioris éventuels quant au recrutement de personnes handicapées. Nous devons donc ouvrir nos formations aux étudiants handicapés. Notre projet, baptisé EEAH (Enseignement de l'Electronique Accessible aux étudiants Handicapés), consiste à édifier une base d'informations qui rendra plus facile l'application de notre démarche dans d'autres universités. La mutualisation des connaissances acquises au sein d'un site web dédié permettra de s'enrichir des expériences à travers la France entière. La communication autour de ce site permettra aux industriels de découvrir des applications concrètes de la mise en accessibilité d'un poste de travail dans le cadre des activités de l'EEA. Nous avons réalisé cette année une adaptation d'un poste de travail au sein du département Mesures Physiques de l'IUT de l'Université Bordeaux 1. Cette opération pilote de notre projet, présentée dans cet article, s'est appuyée sur la présence d'un étudiant tétraplégique au sein de ce département.
\end{abstract}

Mots clés : maquettes adaptées pour personnes handicapées, mutualisation de connaissances et de moyens, information des industriels sur le handicap, aides techniques, interfaces Homme-Machine.

\section{INTRODUCTION}

La Cellule PHASE [1] [2] a pour rôle d'ouvrir les formations dispensées à l'université Bordeaux 1 à des étudiants (artistes confirmés, personnes en situation de handicap, personnes en situation de longue maladie et sportifs de Haut Niveau) qui ne pourraient pas y accéder par suite d'une incompatibilité d'emploi du temps avec des activités extra-universitaires (répétitions ou concerts, séances de soins ou de kinésithérapie, entraînements ou compétitions,...) ou d'inaccessibilité physique. L'encadrement proposé à l'étudiant PHASE lui offre un accompagnement de sa scolarité avec la possibilité d'aménager sa scolarité dans le temps (2,3 ou 4 ans pour un DUT par exemple), de bénéficier de cours de compensation individuels ou encore d'effectuer des aménagements techniques pour les étudiants en situation de handicap (tiers temps, secrétaire personnel...). Dans le cadre de cette dernière mission, une réflexion a été entamée au sein de l'équipe PHASE en collaboration avec des enseignants des départements Génie Electrique et Informatique Industrielle et Mesures Physiques afin de permettre aux étudiants handicapés d'avoir la possibilité de réaliser les mêmes travaux pratiques que leurs camarades. Cela passe par un travail d'adaptation du poste de travail de l'étudiant et des maquettes de travaux pratiques.

Les métiers liés aux formations de l'EEA (Electronique, Electrotechnique, Automatisme) par exemple font de plus en plus appel à des outils qui permettent de créer des interfaces Hommes-Machines. Les actions physiques complexes nécessaires à ces métiers se réduisent considérablement ce qui rend ces métiers accessibles à des personnes handicapées. Au-delà de notre mission de formation qui doit refléter cette évolution, nos universités ont aussi une mission d'information en direction des industriels afin de réduire leurs aprioris éventuels quant au recrutement de personnes handicapées. Nous devons donc ouvrir nos formations aux étudiants handicapés. Pour cela un travail est déjà entamé qui vise à améliorer l'accessibilité des locaux aux personnes handicapées. Il nous faut aussi adapter certains de nos postes de travail de travaux pratiques pour les rendre utilisables par nos étudiants handicapés.

Nous avons entamé cette démarche au sein de la cellule PHASE. Notre projet, baptisé EEAH (Enseignement de l'Electronique Accessible aux étudiants Handicapés), consiste à édifier une base d'informations qui rendra plus facile l'application de notre démarche dans d'autres universités. La mutualisation des connaissances acquises au sein d'un site web dédié permettra de s'enrichir des expériences à travers la France entière. La communication autour de ce site permettra aux industriels de découvrir des applications concrètes de la mise en accessibilité d'un poste de travail dans le cadre des activités de l'EEA. Cette base de connaissance regroupera par exemple des études comparatives du prix de certains équipements (cartes d'interface, souris 
adaptées, attelles ...) des solutions de maquettes de TP (Travaux Pratiques) adaptées (accès aux points de mesure facilités, commutation de composants, sondes de mesure adaptées, expériences pédagogiques ...) et des informations sur les organismes qui peuvent participer au financement de l'achat de ces équipements (AGEFIPH en milieu professionnel [8], DDTEFP [9], FIPHFP pour la fonction publique [12] ...).

Nous avons réalisé cette année une adaptation d'un poste de travail de TP au sein du département Mesure Physique de l'IUT (Institut Universitaire de technologie) de l'Université Bordeaux 1. Cette opération pilote de notre projet, présentée dans cet article, s'est appuyée sur la présence d'un étudiant tétraplégique au sein du département.

\section{PRESENTATION DU TP ET OBJECTIFS PEDAGOGIQUES}

Le TP (proposé aux étudiants par C. Bernou, JM Pham, S. Saïghi et T. Zimmer) s'inscrit dans le cursus de $3^{\circ}$ semestre du DUT Mesures Physiques de l'université Bordeaux 1. L'objectif pédagogique de ce TP est l'utilisation de la détection synchrone afin de réaliser un impédancemètre. Les étudiants, regroupés en binômes, débutent par deux séances qui doivent leur permettre de comprendre et maîtriser les différents aspects de la mise en œuvre d'une conversion analogique-numérique. Puis ils disposent de deux séances pour réaliser le montage complet de l'impédancemètre et de deux séances pour caractériser ce dernier, effectuer des mesures et comparer leurs résultats avec des impédancemètres du commerce.

A travers ces TP les étudiants mettent en application des notions telle qu'une chaîne de mesure. Les différentes parties de cette dernière (capteur, déphaseur, multiplieurs et filtres) sont abordées et réalisées.

Le programme de travail proposé aux étudiants pour la réalisation et le test du montage est présenté dans le tableau 1 (extrait du fascicule de TP) :

\begin{tabular}{|c|}
\hline Séance 1 : \\
- Comprendre la détection synchrone ; de \\
-Comprendre le montage de \\
l'impédancemètre ; \\
-Réaliser le capteur avec un AOP monté \\
en inverseur ; \\
-Réaliser et caractériser le déphaseur ; \\
-Câbler et caractériser les multi- \\
plieurs ; \\
\hline -Réaliser et caractériser les filtres ; \\
-Effectuer des mesures en fonction de \\
différents composants (résistance, \\
self, condensateur) et en fonction de \\
la fréquence du signal ; \\
-Comparer avec un impédancemètre \\
du commerce ; \\
-Tests.
\end{tabular}

Tableau 1 : Programme des séances de TP
Les études théoriques seront menées par les deux étudiants du binôme. La réalisation des câblages sera effectuée par l'étudiant associé à l'étudiant handicapé en respectant une annexe au fascicule de TP indiquant les modifications apportées à la plaquette pour permettre à son binôme d'effectuer les mesures. Ces modifications, présentées dans cet article, permettent à l'étudiant handicapé de réaliser l'ensemble des actions présentées en gras dans le tableau 1. Pour atteindre cet objectif l'étudiant doit pouvoir manipuler l'oscilloscope, le générateur de signaux, la sonde de mesure et les composants à tester. Les principaux problèmes rencontrés et les principales solutions apportées sont présentés dans les paragraphes suivants.

\section{EXEMPLE DE PROBLEMES REN- CONTRES}

Lors de la première séance, nous avons eu un entretien avec l'étudiant afin d'identifier la mobilité qu'il possède pour centrer nos recherches. Cette étape est importante et spécifique à l'étudiant et à son handicap (moteur, visuel ...). Cette expertise peut s'appuyer sur l'intervention de professionnels extérieurs (ergothérapeute...).

\subsection{Accès au poste de travail}

Un problème d'accessibilité au poste de travail a été tout de suite mis en évidence. La table étant trop basse, il ne peut pas manipuler dans de bonnes conditions en raison de la difficulté d'accès avec son fauteuil roulant électrique (figure 2). Ce point peut être facilement solutionné par l'achat d'une table de travail adaptée ou la fabrication de cales de surélévation beaucoup moins onéreuses.

\subsection{L'utilisation des appareils}

Le principal appareil de mesure utilisé est un oscilloscope numérique (référence: Agilent 54621A [4]). L'étudiant a des difficultés pour l'allumer. De plus, il ne peut effectuer aucun réglage de balayage et d'échelle qui permet de visualiser un signal convenablement (figure 1).

Le TP utilise aussi un générateur de signaux (GBF, référence : Agilent 33220A [5]) et un multimètre (référence: Sefram 7210 [6]) que l'étudiant ne peut pas régler. 


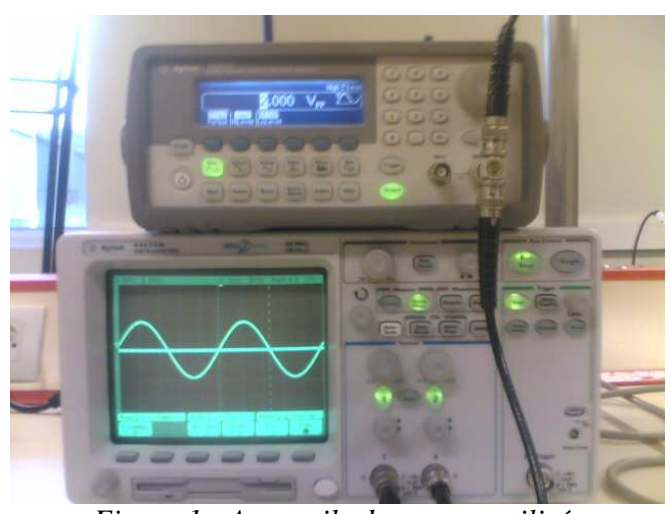

Figure 1 : Appareils de mesure utilisés

\subsection{La carte électronique}

Les TP d'électronique utilisent une carte qui rassemble les différents circuits sur lesquels l'étudiant doit faire des mesures. Malheureusement les points de mesure sont difficilement accessibles pour cet étudiant qui ne possède pas de motricité au niveau des mains. Dans ces conditions, il ne pourra pas effectuer de relevé de mesures.

\subsection{Utilisation de la sonde de mesure}

Pour effectuer les mesures, l'étudiant doit, de plus, utiliser une sonde de mesure (référence : Agilent 10074C [7]). Le problème réside ici dans le maniement de la sonde tout en effectuant les mesures. Malheureusement, l'étudiant ne peut pas prendre correctement en main la sonde (figure 2).

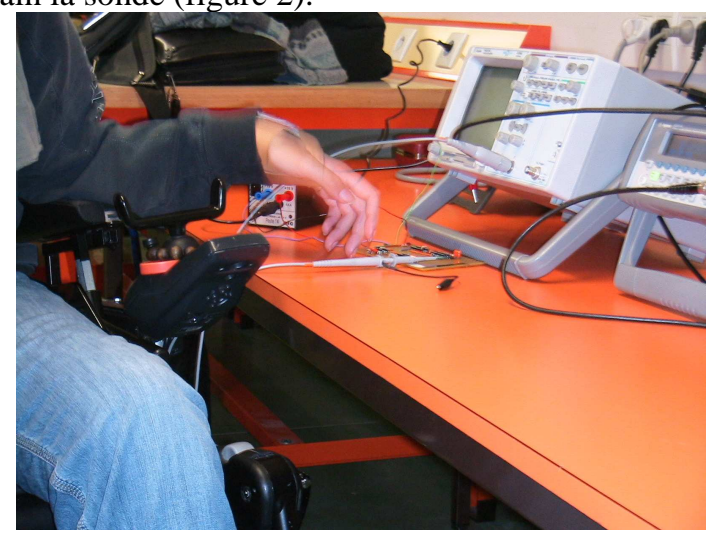

Figure 2 : Accès au poste de travail et prise en main de la sonde de mesure

\subsection{Changement des composants sur la plaquette}

L'objectif de ce banc de mesure est de mesurer l'impédance de divers composants. L'étudiant a de grandes difficultés pour changer les composants de la plaquette sur lesquels une analyse doit être faite.

\section{EXEMPLES DE SOLUTIONS APPORTEES}

\subsection{Commande des appareils}

La solution retenue a été l'achat d'un oscilloscope et d'un générateur de signaux intégrés qui peuvent être connectés par liaison USB à un ordinateur (DS1M12) [2][10].

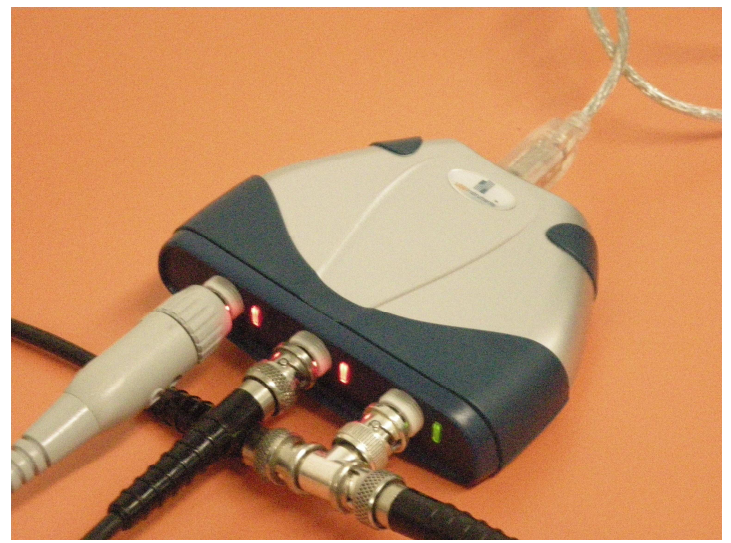

Figure 3 : L'oscilloscope USB (DS1M12 "Stingray")

Cette solution (figure 3), d'un faible coût (265 euros) est simple et rapide à mettre en place. Elle permet de disposer d'un oscilloscope, d'un GBF et d'un multimètre commandés par un ordinateur portable.

L'étudiant peut alors régler les paramètres du générateur de signaux (figure 4).

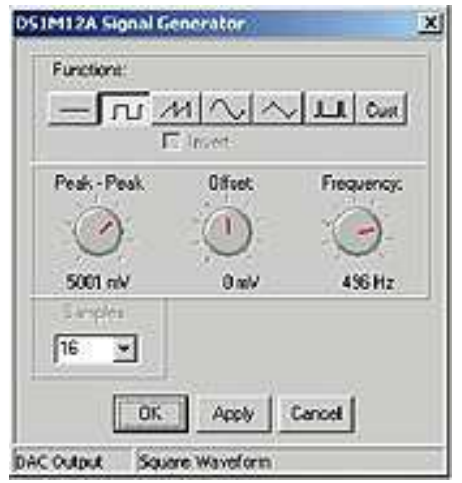

Figure 4 : Commande du GBF

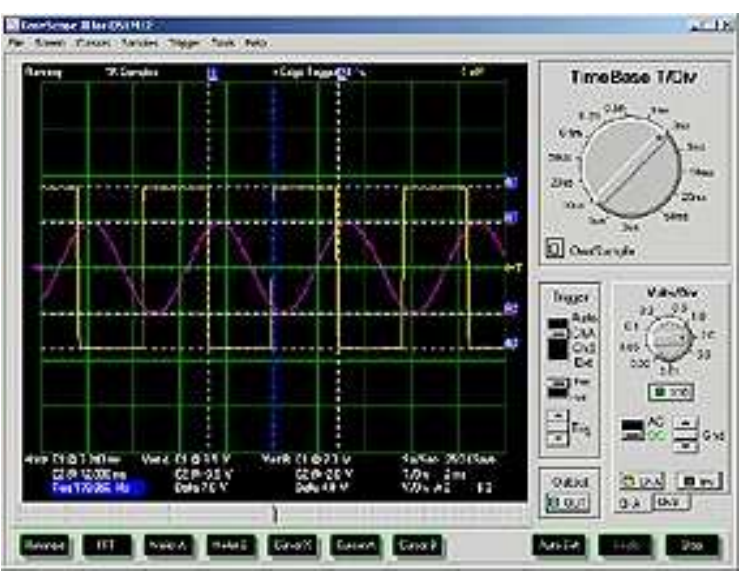

Figure 5 : Interface de l'oscilloscope du DS1M12

L'utilisation de cet appareil lui permet aussi, via l'ordinateur, de régler et de déterminer les différentes caractéristiques de la mesure, à savoir le déphasage 
entre deux signaux, la fréquence du signal ou son amplitude et de relever les courbes nécessaires au TP (figure 5).

Il peut aussi utiliser le DS1M12 en multimètre (figure 6) pour mesurer des tensions ou des courants.

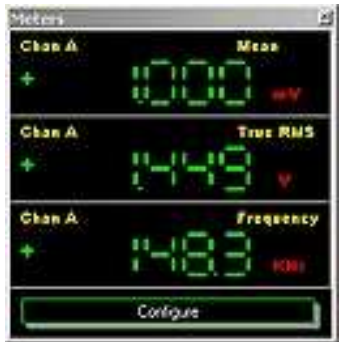

Figure 6 : Multimètre disponible à l'aide du DS1M12

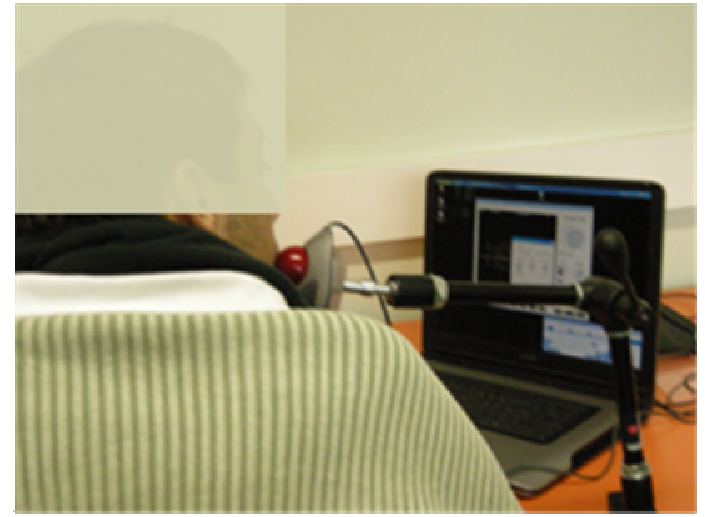

Figure 7 : Utilisation d'une souris track-ball

Notre étudiant a alors des difficultés pour utiliser l'ordinateur sans l'utilisation d'une souris adaptée. Il existe différents modèles de souris adaptées pour personnes handicapées. Nous pouvons trouver des souris contacteur, des track-ball menton, des émulateurs de souris infrarouge (HeadMouse extrême® [11] par exemple) ou encore l'utilisation de click virtuel par la mise en place de capteur. Dans notre cas l'étudiant utilise une souris track-ball menton placé au bout d'un bras articulé, qu'il dirige avec son menton (Figure 7). Il peut alors contrôler les équipements à l'aide de l'interface du DS1M12 installé sur son ordinateur.

L'utilisation du clavier virtuel de Windows ou de logiciel libre (Click-N-Type) lui permet de prendre des notes durant le TP. Il est à noter que, par un phénomène de compensation, la personne handicapée peut développer une aptitude insoupçonnée pour le maniement de ces outils.

Pour les étudiants malvoyants l'utilisation de l'outil «loupe » de Windows et le changement de résolution de l'ordinateur permettent une utilisation correcte de l'interface.

\subsection{Prise de mesure}

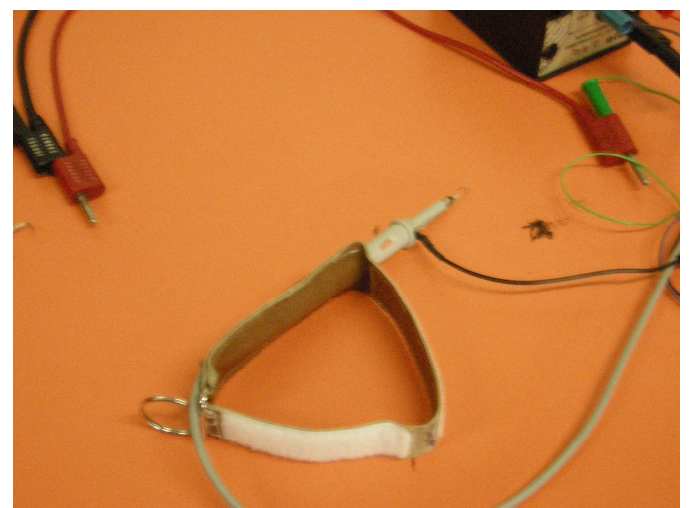

Figure 8 : Bracelet de préhension de la sonde de mesure

La solution de la prise de la sonde peut passer par l'utilisation d'un bracelet développé spécifiquement pour cette application, sur lequel la sonde est fixée (figure 8).

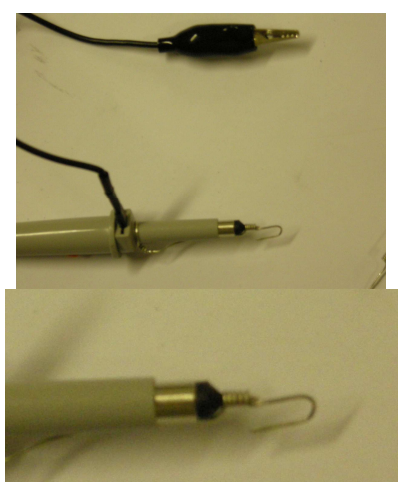

Figure 9: Adaptation de la sonde de mesure

Sur la plaquette, des anneaux ont été ajouté afin de faciliter l'accès aux points de mesure.

L'ajout d'un embout métallique en forme de crochet au bout de la sonde permet à l'étudiant de l'accrocher aux anneaux de mesure (Figure 9). Une étude a été effectuée afin de s'assurer que la présence de cet «embout» ne perturbe pas les mesures.

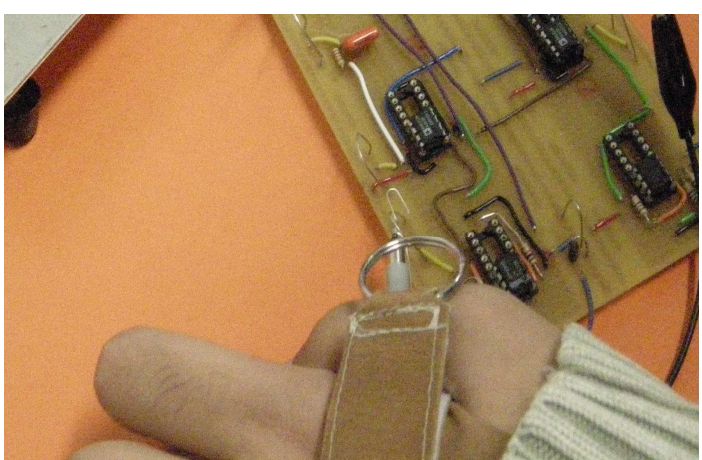

Figure 10 : Prise de mesure

La sonde est fixée aux points de mesure désirés, et l'élève peut alors se concentrer sur le reste de la mesure (Figure 10). 


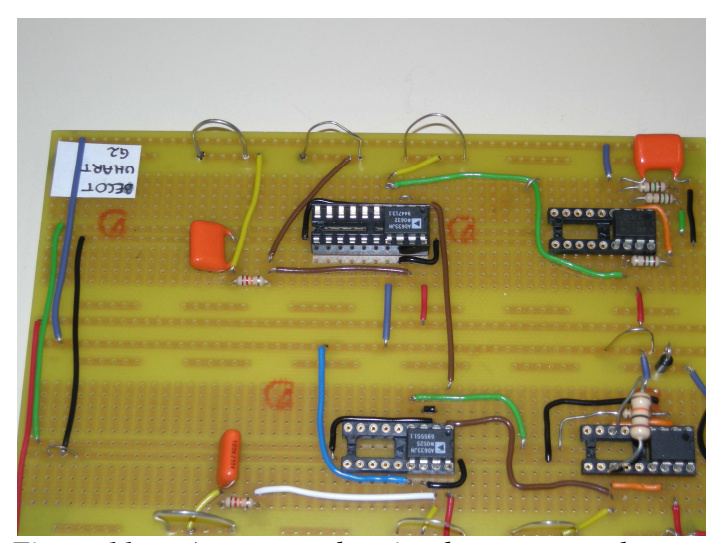

Figure 11: «Anneaux » de prise de mesure sur la carte

\subsection{Changement des composants}

Afin de permettre à l'étudiant d'effectuer des mesures sur différents composants ceux-ci ont été montés sur des supports que l'étudiant peut manipuler (Figure 12). Ces supports sont ensuite placés par l'étudiant sur une plaquette reliée à la maquette principale (Figure 13).

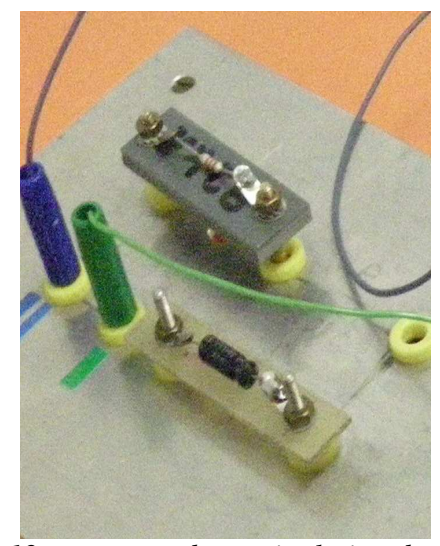

Figure 12 : supports de manipulation des composants

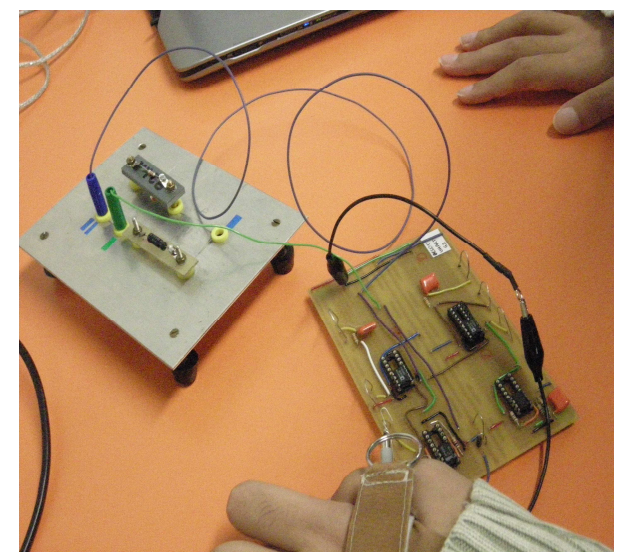

Figure 13 : Maquette de TP complète

\section{CONCLUSION}

Le travail réalisé a permis à l'étudiant handicapé d'effectuer des manipulations et des prises de mesure. Il pourra ainsi atteindre une grande partie des objectifs pédagogiques de ce TP en le réalisant au même titre que ses camarades. La réussite de cette expérience va nous conduire à proposer régulièrement des sujets de projets tuteurés aux étudiants de DUT afin d'enrichir notre base de données et notre bibliothèque de matériel. Le matériel acheté ou développé dans le cadre de ce projet est destiné à être mis à disposition des filières de formation de l'université de Bordeaux 1 qui accueillent un public handicapé. Une communication envers les entreprises et le grand public (fête de la science ...) va être menée. Un dossier d'information a déjà été réalisé au sujet de l'adaptation de ce poste de travail. L'étudiant handicapé pourra l'utiliser lors de sa recherche d'emploi. Ce dossier présente les aides techniques que nous avons étudiées afin de démontrer que l'emploi d'une personne handicapée dans les métiers de l'EEA n'est pas une utopie.

\section{Bibliographie}

[1] http://www.u-bordeaux1.fr/phase/

[2] http://www.iut.u-bordeaux1.fr/services/cp/cp.htm

[3] http://www.usb-instruments.com/oscillo stingray.html

[4] http://www.home.agilent.com/agilent/product.jspx?p $\underline{\mathrm{n}=54621 \mathrm{~A}}$

[5]http://www.home.agilent.com/agilent/product.jspx?c $\mathrm{c}=\mathrm{US} \& \mathrm{lc}=\mathrm{eng} \& \mathrm{nid}=-536902257.536883183$

[6]http://www.sefram.com/www/HauteResolution/inde $\underline{x . a s p}$

[7] http://www.home.agilent.com/agilent/product.jspx?n $\underline{\mathrm{id}=-536902770.536880407 .00 \& \mathrm{cc}=\mathrm{US} \& \mathrm{lc}=\mathrm{eng}}$

$$
\text { http://www.agefiph.fr/ }
$$

[9],http://www.handicap.gouv.fr/rubrique.php3?id_rubr $\underline{\text { ique }=40}$

[10] http://www.lextronic.fr/P899-oscilloscope-usbds1m12.html

[11] http://www.orin.com/access/headmouse/index.htm

[12] http://www.fiphfp.fr/

[13]Fascicule de TP «Application de la détection synchrone à la mesure d'impédance ». C. Bernou, JM Pham, S. Saïghi, T. Zimmer 